\title{
The Role of Raman Spectroscopy in Differentiating Grades of Dysplasia in Barrett's Oesophagus: Is It Currently Good Enough?
}

\author{
Upchurch $E^{1,2^{*}}$, Isabelle $M^{3}$, Lloyd $G R^{4}$, Kendall $C^{1}$, Stone $N^{5}$ and Barr $H^{1,2}$
}

${ }^{1}$ Biophotonics Research Unit, Gloucestershire Royal Hospital, Gloucester, UK

${ }^{2}$ Department of Upper GI Surgery, Gloucestershire Royal Hospital, Gloucester, UK

${ }^{3}$ Renishaw PIc, Gloucestershire, UK

${ }^{4}$ Phenome Centre Birmingham, School of Biosciences, University of Birmingham, Gloucester, UK

${ }^{5}$ College of Engineering, Mathematics and Physical Sciences, University of Exeter, Exeter, UK

\begin{abstract}
Many cancers, including oesophageal adenocarcinoma, develop from tissue that becomes increasingly dysplastic, however, these changes are often difficult to identify by histopathological changes. If these changes can be diagnosed at an early stage, this has the potential to improve management, and outcome, for these patients. Raman spectroscopy has the potential to be such a tool, with the ability to diagnose pathological changes through the elucidation of spectral biomarkers that are associated with biochemical changes that are the result of disease progression.
\end{abstract}

\section{Keywords}

Dysplasia, Barrett's oesophagus, Raman spectroscopy, Oesophageal adenocarcinoma

\section{Introduction}

Many cancers, including oesophageal adenocarcinoma, develop from tissue that becomes increasingly dysplastic. The outcome, in terms of reduced mortality and morbidity, is vastly improved when diagnosis occurs when the cancer is at an early stage, remaining localised to the tissue from which it developed. The outcomes are even better when cancer has not yet developed and the pre-cancerous changes can be treated. The difficulty with this approach, nevertheless, is that these changes are difficult to identify. This paper will discuss oesophageal cancer and its precancerous precursor and the role of Raman spectroscopy in identifying these pathological changes.

\section{Oesophageal Adenocarcinoma}

Oesophageal adenocarcinoma has been increasing in incidence since the 1970 's; with $43 \%$ more cases being diagnosed. The survival rates remain poor with an overall 5-year survival of $15.1 \%$ [1]. Survival rates are highest $(\sim 40 \%)$ for patients with disease that is confined to the oesophagus at the time of diagnosis [1] as these patients have the potential to be cured by surgical resection of the oesophagus. This, however, is the smallest group of patients as symptoms typically only develop when the cancer has become larger and already metastasised. This is a significant undertaking associated with significant morbidity and impact on quality of life.

Patients with small foci of cancer which is limited to the mucosa, the innermost layer of the oesophagus, can be treated by endomucosal resection which removes the cancer only, without having to resect the oesophagus. This, obviously, is less invasive. Identification of patients

${ }^{*}$ Corresponding author: Emma Upchurch, Biophotonics Research Unit, Department of Upper GI Surgery, Gloucestershire Royal Hospital, Great Western Road, Gloucester, GL13NN, UK, E-mail: em_upchurch@hotmail.com

Received: November 19, 2017: Accepted: February 15, 2018: Published: February 17, 2018

Copyright: (C) 2018 Upchurch E, et al. This is an open-access article distributed under the terms of the Creative Commons Attribution License, which permits unrestricted use, distribution and reproduction in any medium, provided the original author and source are credited. 
with very early cancer that can be treated by endomucosal resection, or even at risk patients who have not yet developed cancerous changes, will enable the curative treatment of a greater cohort and, hence, improve outcomes from this disease.

\section{Barrett's Oesophagus}

Barrett's oesophagus is an acquired condition, characterised by columnar epithelium (tongues of salmon pink) replacing the squamous epithelium (pale pink mucosa) in the distal oesophagus (Figure 1). This metaplastic change is thought to be an adaptive response to the increased cell loss that is a result of chronic inflammation in this area. The significance of the change, nevertheless, is the potential of the cells to develop dysplastic changes and, in some cases, become cancerous.

Barrett's oesophagus is, thus, recognised as a precursor to oesophageal adenocarcinoma, however, only

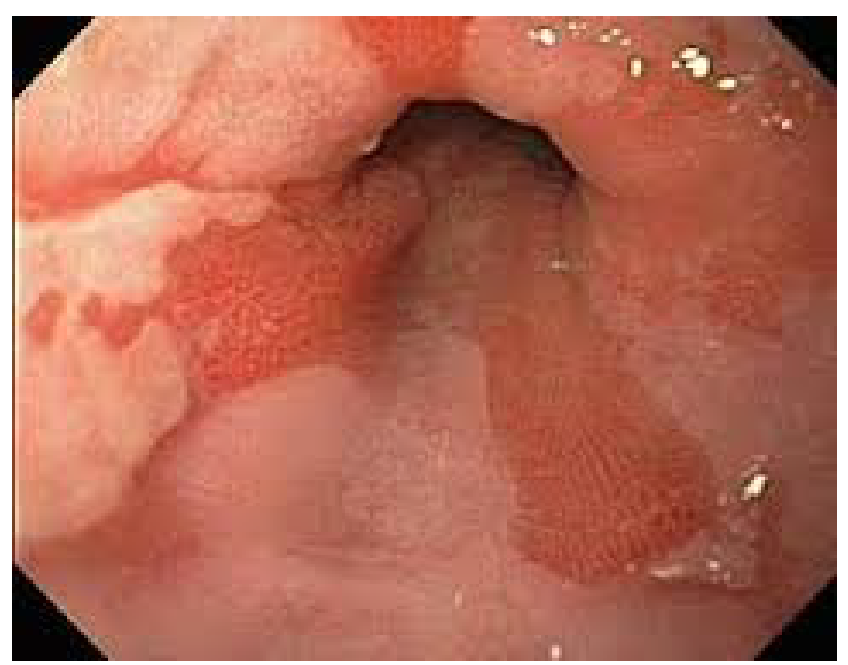

Figure 1: Endoscopic appearance of Barrett's oesophagus (Courtesy of Digestive Health Associates: Southwest Endoscopy Centre). a minority of patients will progress to adenocarcinoma. Two population based studies have shown progression rates of $0.22 \%$ [2] and $0.26 \%$ [3] per year, and the most up-to-date meta-analysis calculated a progression rate of $0.33 \%$ [4] per year. Within the population of patients with Barrett's oesophagus, the greatest risk of progression occurs in patients who have already developed dysplastic changes.

The current protocol for identifying dysplasia is based on microscopic morphological changes that are seen on histopathological analysis of biopsy samples. The changes of dysplasia are a continuum with low-grade dysplasia being more similar to normal tissue and high-grade dysplasia being further along on the continuum to adenocarcinoma (Figure 2). The features of dysplasia include a complex array of changes and, thus, it is hardly surprising that a high degree of intra-and inter-observer variability exists in the diagnosis of dysplasia [5-7]. A better means for identifying the presence and grade of dysplasia is, thus, urgently required in the quest to improve the outcomes for oesophageal adenocarcinoma and provide appropriate risk stratification for patients with Barrett's oesophagus. Ideally, a means of identifying which patients will go on to develop adenocarcinoma would enable surveillance and treatment of this important cohort.

\section{Vibrational Spectroscopy}

Molecules are composed of atoms connected by chemical bonds. Each bond has a characteristic vibrational energy. When illuminated with electromagnetic radiation, the molecule enters a virtual energy state, resulting in the vibration of the chemical bonds. The electrons are excited into a higher energy level by absorption of a photon, usually from the electromagnetic radiation in the form of a laser light source. When the electron then decays back to a lower energy level, a scattered photon is emitted and it is the difference in energy of the
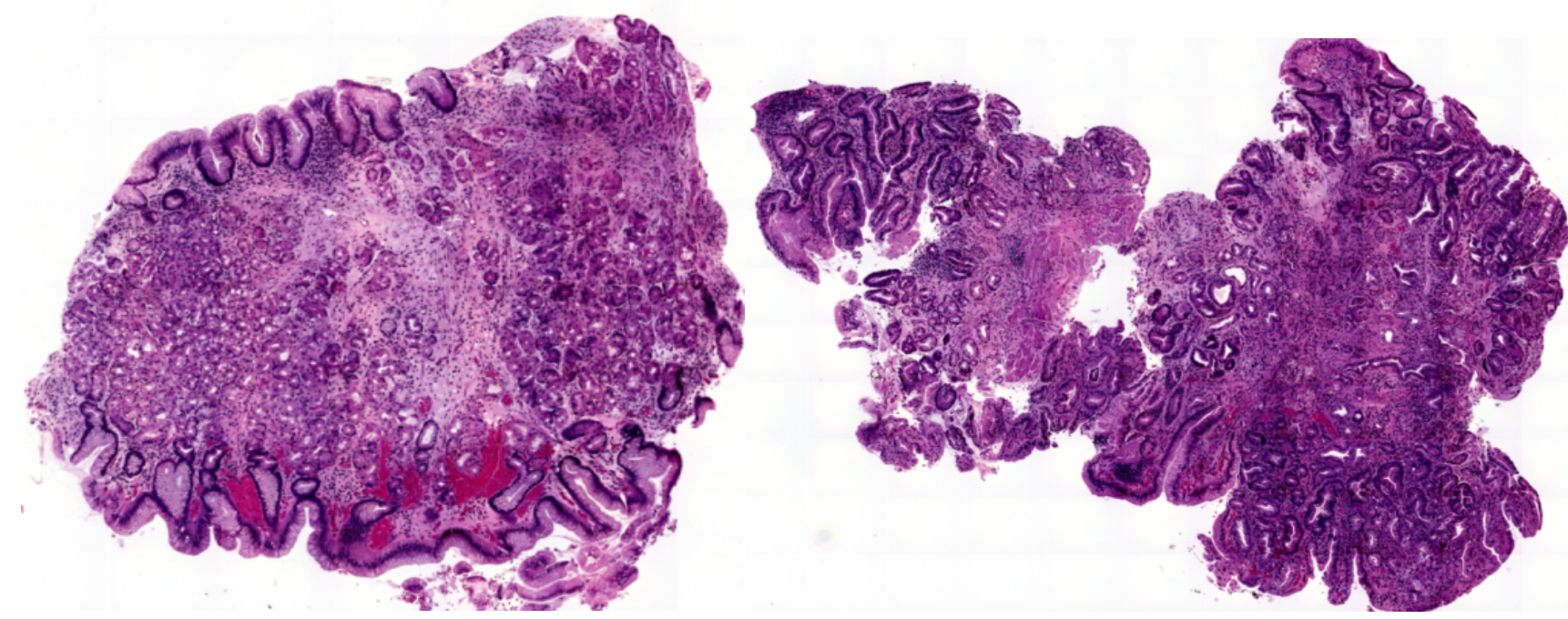

Figure 2: Low Grade (left image) and High Grade Dysplasia (right image) in Barrett's Oesophagus. 
non-scattered and scattered photons that corresponds to the energy of the molecular vibration.

The inelastic scattering of light was named after one of its discoverers, Sir Chandrasekhara Venkata Raman [8]. The detection of the scattered photons can provide a spectrum of Raman peaks based on the bonds present in the molecule and tissue sample being analysed. This enables a wealth of information regarding the composition of the molecule to be obtained. Subtle differences in different tissue samples, such as those in low-grade and high-grade dysplasia would, therefore, be expected to be detected by this method. More detailed analysis on vibrational spectroscopy is beyond the scope of this paper, however, suggested reading is included in the reference list [9-11].

\section{Raman Spectroscopy in the Oesophagus}

For many years, in ex-vivo studies, Raman spectroscopy has been shown to be able to discriminate between different pathology groups in the oesophagus, including those changes of Barrett's oesophagus [12-14]. For example, eight different pathology groups, including different grades of dysplasia, were shown to be able to be discriminated when snap-frozen biopsy samples were analysed by Raman spectroscopy [15]. Following on from this, a significant volume of work has focused on using real-time, in-vivo measurements [16-20], however, further work into the discrimination of low-grade dysplasia in particular is needed to improve detection rates and aid the understanding of its natural history.

As discussed, only a small number of patients with Barrett's oesophagus continue towards malignancy. This number is highest for patients who already have high-grade dysplasia. Low-grade dysplasia remains, in some respects, a mystery as many patients will not progress further and may even regress. The most recent meta-analysis calculated the risk of progression of lowgrade dysplasia to adenocarcinoma as $0.33 \%$ per year [4]. It may be that there are patients with pertinent changes of low-grade dysplasia who are more likely to progress and the more detailed analysis that Raman spectroscopy provides, may aid in understanding what these features are and identify which patients are at risk.

\section{Differentiating between Grades of Dysplasia: Purpose of the Study}

The management of patients with Barrett's oesophagus varies considerably depending on the grade of dysplasia, from that of active surveillance through to oesophagectomy. The ability to accurately determine the grade of dysplasia is, thus, of paramount importance. Recent studies using both Raman point spectroscopy and Raman mapping has demonstrated the ability of this technology to differentiate samples of low-grade dyspla- sia from samples of high-grade dysplasia and adenocarcinoma $[14,15]$. The purpose of this study was to ascertain if these differences could be detected using point spectra measurements, which enable rapid acquisition of data, and if this gave the same detail of data as that of Raman map measurements.

\section{Methods}

Point spectra measurements collect a single static point of the tissue sample in a rapid time frame. A longer time-frame allows a more detailed analysis. For this work, each sample was measured at approximately 60 points with each measurement taking 30 seconds. Multiple measurements ensure that a comprehensive picture of the overall sample is built.

This study used archived samples which had been stored in paraffin tissue blocks. Tissue sections of $7 \mu \mathrm{m}$ thickness were prepared on calcium fluoride slides. Due to the significant peaks that paraffin produces on Raman spectroscopy analysis, the samples were deparaffinised, using Hexane $\left(\mathrm{C}_{6} \mathrm{H}_{14}\right)$, prior to measurement. The samples were then stored at room temperature prior to measurement.

\section{Results}

In this study only a very small data set was collected and analysed. 10 samples each of low-grade dysplasia, high-grade dysplasia and adenocarcinoma were measured using the Renishaw System 1000 at the Biophotonics Research Unit at Gloucestershire Royal Hospital, resulting in a total sample size of 30 . A significant degree of overlap is seen between the samples of different pathology types (Figure 3). This emphasises that each tissue type is not wholly unique, instead there are more subtle changes that indicate the underlying tissue type. The changes are most apparent in adenocarcinoma as depicted in Figure 3, yet the changes of low-grade and high-grade dysplasia are more closely aligned, hence, the higher overlap of these tissue types. The small differences between samples of low-grade dysplasia and those of high-grade dysplasia and adenocarcinoma is reflected by a specificity of 0.70 and a sensitivity of 0.84 when differentiating these tissue types.

Point spectra measurements have the advantage of rapid data acquisition which makes it attractive for in-vivo use, however, it may suffer from missing vital areas of diagnostic criteria, reducing the specificity of the technique.

The same samples were also analysed using Raman mapping measurements (using the Renishaw RA800 Series bench top Raman system at the Biophotonics Research Unit in Gloucestershire Royal Hospital). Raman mapping measures a larger area of the tissue sample, however, this increase in information comes at a cost of significant time 


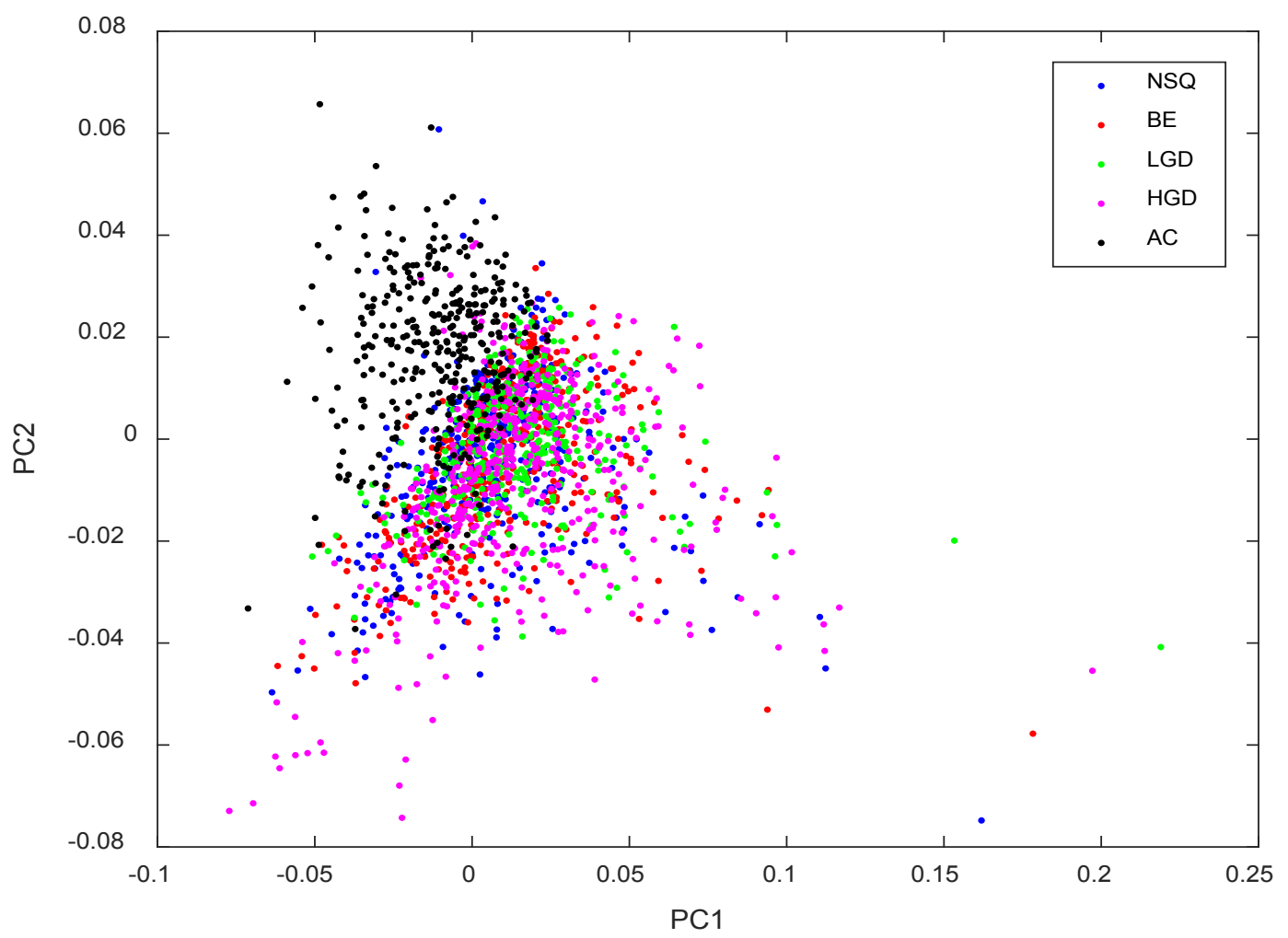

Figure 3: Scatter plot of Raman Point Spectra Measurements (NSQ = Normal Squamous Tissue; BE = Barrett's Oesophagus without dysplasia; LGD = Barrett's oesophagus with Low-Grade Dysplasia; HGD = Barrett's oesophagus with High-Grade Dysplasia; $A C=$ Oesophageal Adenocarcinoma).

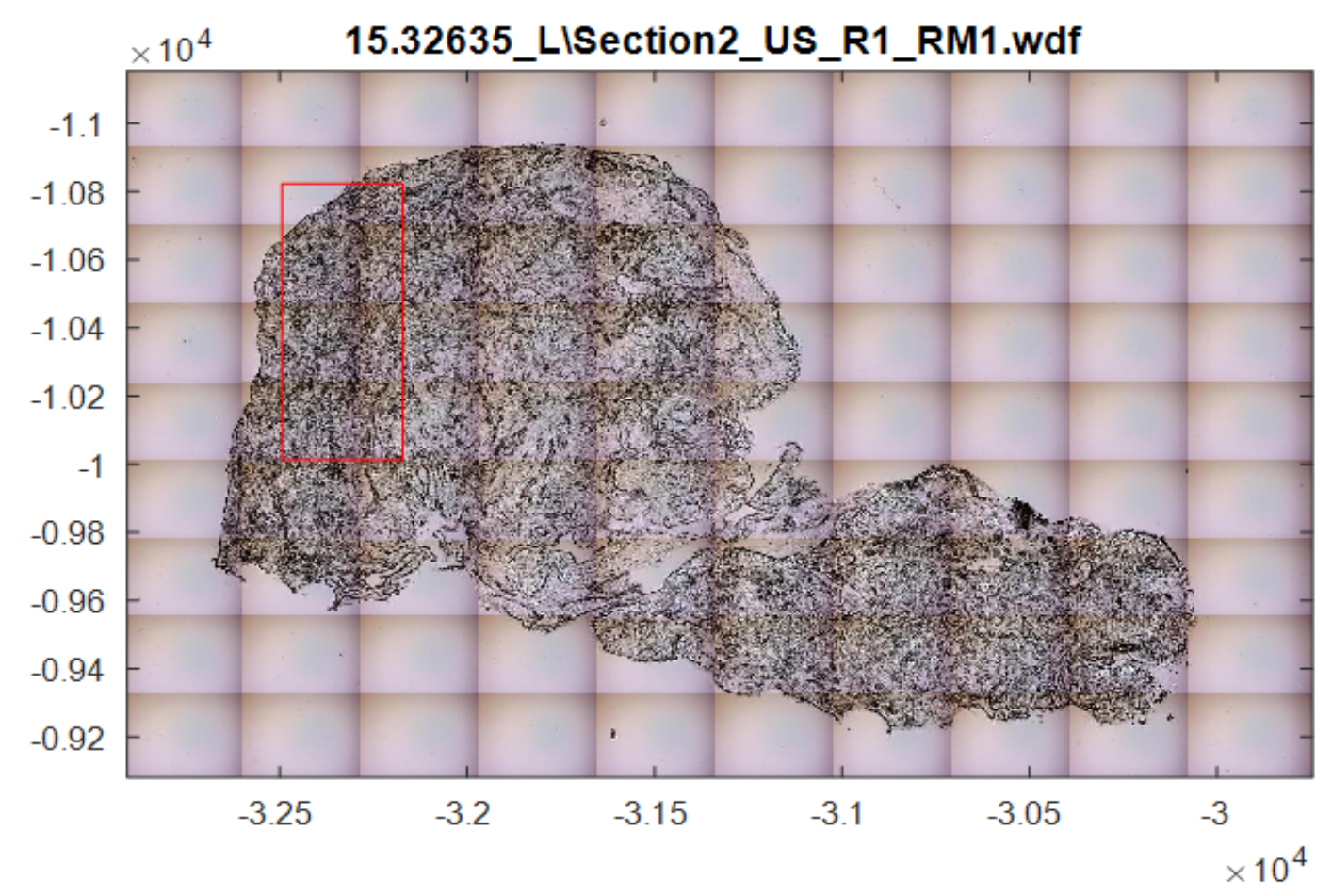

Figure 4: Example of area analysed using Raman mapping.

to map the sample, often many hours. A forceps biopsy of the oesophagus is approximately $2 \mathrm{~mm}$ diameter. The area highlighted in Figure 4, an area of approximately $0.25 \mathrm{~mm}$, took 31 minutes to analyse. This would obviously prevent the use of mapping in in-vivo samples. The differentiation of low-grade dysplasia from that of high-grade dysplasia and adenocarcinoma using mapping samples had a specificity of 0.51 and a sensitivity of 0.88 . 
The reduction in specificity with mapping results compared to point spectra is somewhat unexpected as one would expect that the increased data would improve accuracy. The changes of low-grade and high-grade dysplasia are, however, not uniform across a given biopsy sample and, thus, measurement of the complete sample is likely to include normal areas of tissue which are the same in both samples. This concept is important to consider when applying spectroscopy to tissue samples as it is not the average over the whole sample, but actually even very small areas of the highest degree of dysplasia that are the most important. Diagnostic models will need to be able to account for this, particularly as, in-vivo, it would be impossible to know which areas are included as they are macroscopically identical.

\section{Discussion}

The Role of Spectroscopy in identifying small differences in tissue samples: What if it is better than the Gold Standard?

As discussed, Raman spectroscopy has been shown to accurately identify cancerous tissue from that of non-cancerous tissue in the oesophagus $[12,13]$. The more subtle changes of dysplasia in Barrett's oesophagus can also be differentiated, however, the specificity and sensitivity is lower [14,15].

Low-grade dysplasia is likely to represent a heterogeneous group of tissues. Some patients with low-grade dysplasia will never progress to adenocarcinoma and may even regress, whereas, others will continue along the spectrum to malignancy. As well as possible genetic changes that separate these different cohorts of patients, there may be differences in the tissues that are not detectable by histopathology, yet are by Raman spectroscopy. By analysing these potentially disparate groups as a single group may be detrimental to our own results as significant intra-group differences exist. Using proteomics and genomics in combination with Raman spectroscopy to train the models, however, may enable a more specific molecular pathology status to be assigned to certain spectral datasets, thereby, revealing specific Raman spectral biomarkers.

Raman spectroscopy is likely to be able to detect greater differences than those that we can appreciate by the current means of histopathology. One of the challenges, therefore, is to be able to ascertain which differences are real and which may be error. One of the ways in which this could be elicited would be to analyse samples retrospectively once knowledge of their clinical progression is known. This would enable different cohorts of low-grade dysplasia based on their progression to be analysed and differentiated. As it is primarily the risk of progression in low-grade dysplasia that is of paramount importance, being able to identify this using Raman spectroscopy would provide the most beneficial information that clinician's crave.

\section{Challenges in Raman Spectroscopy}

Raman spectroscopy has, thus far, failed to make the transition into clinical practice. Part of this is due to the lack of clarity as to where this technology would provide the most benefit, as well as the lack of large scale randomised trials highlighting and proving its value. Obviously, if this technology can provide information that is unattainable through histopathology (as discussed above), then its value would be self-explanatory. Until this point, the possible roles of Raman spectroscopy are in automated screening and real-time, in-vivo sampling and analysis.

The current protocol for patients undergoing surveillance endoscopy with Barrett's oesophagus is to take quadrantic biopsies at $2 \mathrm{~cm}$ intervals in the segment of Barrett's. A patient with a $6 \mathrm{~cm}$ segment of Barrett's will, therefore, have 12 biopsies. The analysis of these samples places a considerable burden on the time and resources of the Histopathology department.

It has previously been shown that $2 \mathrm{~mm}$ diameter sections, mapped over 30-90 minutes, provide sufficient information to enable the discrimination of pathology [21]. The point spectra measurements used in this study took a comparable time-frame (60 measurements of 30 seconds). Given the number of samples that require analysis, this remains a substantial time period.

If the time period could be reduced, whilst still maintaining the specificity and sensitivity required, and this process could be automated, then this would provide a means of screening the samples. Samples of normal oesophageal epithelium and samples of Barrett's oesophagus without dysplasia could be screened and would, therefore, require no further review. Samples with dysplasia could then have more detailed analysis, in the form of both Histopathology review and more detailed Raman spectroscopy in order to provide an accurate diagnosis of the degree of dysplasia present.

A number of fibre-optic Raman probes have been developed for use with commercial endoscopes [16-20]. This technology would allow real time diagnosis of dysplasia at the time of the endoscopy and, thus, could be used to guide biopsy sampling sites. Currently biopsies are taken at random sites and it has been shown that areas of dysplasia and even adenocarcinoma are missed [12].

Real time sampling would need the probe to maintain contact with an oesophagus that is in constant peristalsis and, thus, a rapid time-frame would be required. In addition, a high specificity would be essential so areas are not wrongly assumed to be normal and not biopsied. 
Recent work by Bergholt, et al. 2011 [19] and 2014 [20] using an in-vivo probe demonstrated high specificity of $>90 \%$ in identifying oesophageal adenocarcino$\mathrm{ma}$ and areas of dysplasia in a timeframe of 0.5 seconds. These studies, nevertheless, focused on abnormal areas of the oesophagus. The majority of Barrett's oesophagus, however, appears uniform and the full value of this technology would only be apparent if it can identify low-grade and high-grade dysplasia in macroscopically normal Barrett's oesophagus. Studies are needed which compare this technology with random biopsies to ascertain if in-vivo Raman sampling provides a superior diagnostic yield.

\section{Summary Remarks}

Raman Spectroscopy has shown huge promise and potential in the diagnosis of tissue samples, particularly ex-vivo. There is no doubt that this technology can identify dysplastic and cancerous tissue. This study has echoed previous studies, again demonstrating the differentiation of grades of dysplasia in Barrett's oesophagus. This study demonstrated this using point spectra measurements which enable swift and accurate diagnosis and could, thus, be utilised in-vivo. Nevertheless, these findings do not identify how Raman spectroscopy can take the diagnosis of Barrett's oesophagus and pre-cancerous changes forward, despite showing huge promise and potential.

The main problem is how to utilise this technology in a meaningful and useful way to improve patient care and outcome and, thus far, this dilemma has not been answered. An ideal benefit would be if Raman spectroscopy could identify the small group of patients with Barrett's oesophagus who are likely to progress to adenocarcinoma and, hence, allow treatment of this group. An additional difficulty is, however, that many patients with Barrett's oesophagus are asymptomatic and, thus, unidentified. If biochemical changes that are consistent with pre-cancerous changes are identified, then this could lead to biomarkers, enabling screening technology and, hence, identify these changes in asymptomatic patients.

\section{References}

1. (2013) Cancer Key Facts. Cancer Research UK.

2. Hvid-Jensen F, Pedersen L, Drewes AM, Sørensen HT, Funch-Jensen P (2011) Incidence of adenocarcinoma among patients with Barrett's esophagus. $\mathrm{N}$ Engl J Med 365: 1375-1383.

3. Bhat $S$, Coleman $H$, Yousef $F$, Johnston BT, McManus DT, et al. (2011) Risk of malignant progression in Barrett's Esophagus patients: results from a large population-based study. $J$ Natl Cancer Inst 103: 1049-1057.

4. Desai T, Krishnan K, Samala N, Singh J, Cluley J, et al. (2012) The incidence of oesophageal adenocarcinoma in non-dysplastic Barrett's oesophagus: a meta-analysis. Gut 61: 970-976.

5. Kerkhof M, Van Dekken H, Steyerberg EW, Meijer GA, Mulder AH, et al. (2007) Grading of dysplasia in Barrett's oesophagus: Substantial interobserver variation between general and gastrointestinal pathologists. Histopathology 50: $920-927$.

6. Lee YC, Cook MB, Bhatia S, Chow WH, El-Omar EM, et al. (2010) Inter-observer reliability in the endoscopic diagnosis and grading of Barrett's esophagus: an Asian multinational study. Endoscopy 42: 699-704.

7. Gaddam S, Mathur SC, Singh M, Arora J, Wani SB, et al. (2011) Novel probe-based confocal laser endomicroscopy criteria and inter-observer agreement for the detection of dysplasia in Barrett's esophagus. Am J Gastroenterol 106: 1961-1969.

8. Raman CV (1928) A New Radiation. Indian J Phys 2: 387398.

9. Diem M (2015) Modern Vibrational Spectroscopy \& Micro-Spectroscopy: Theory, Instrumentation and Biomedical Application. ( $1^{\text {st }}$ edn), Wiley-Blackwell.

10. Baker M, Hughes C, Hollywood K (2016) Biophotonics: Vibrational Spectroscopic Diagnostics. IOP Science.

11. Hugh Byrne, Kamila Ostrowska, Haq Nawaz, Jennifer Dorney, Aidan Meade, et al. (2014) Vibrational Spectroscopy: Disease Diagnostics and Beyond. In: M Baranska, Optical Spectroscopy and Computational Methods in Biology and Medicine. Springer Series, Challenges and Advances in Computational Chemistry and Physics.

12. Vieth M, Ell C, Gossner L, May A, Stolte M (2004) Histological analysis of endoscopic resection specimens from 326 patients with Barrett's esophagus and early neoplasia. Endoscopy 36: 776-781.

13. Shetty G, Kendall C, Shepherd N, Stone N, Barr H (2006) Raman spectroscopy: elucidation of biochemical changes in carcinogenesis of esophagus. Br $\mathrm{J}$ Cancer 94: 14601464.

14. Almond L, Hutchings J, Lloyd GR (2014) Endoscopic Raman spectroscopy enables objective diagnosis of dysplasia in Barrett's esophagus. Gastrointest Endosc 79: 37-45.

15. Kendall C, Stone N, Shepherd N, Geboes K, Warren B, et al. (2003) Raman Spectroscopy, a potential tool for the objective identification of classification of neoplasia in Barrett's oesophagus. J Pathol 200: 602-609.

16. Martin G Shim, Brian C Wilson, Eric Marple, Michael Wach (1999) Study of fiber-optic probes for in-vivo medical Raman spectroscopy. Applied Spectroscopy 53: 619-627.

17. Day JC, Bennett R, Smith B, Kendall C, Hutchings J, et al. (2009) A miniature confocal Raman probe for endoscopic use. Phys Med Biol 54: 7077-7087.

18. Huang Z, Teh SK, Zheng W, Mo J, Lin K, et al. (2009) Integrated Raman spectroscopy and trimodal wide-field imaging techniques for real-time in-vivo tissue Raman measurements at endoscopy. Opt Lett 34: 758-760.

19. Bergholt M, Zheng W, Lin K, Ho KY, Teh M, et al. (2011) In-vivo diagnosis of esophageal cancer using image-guided raman endoscopy and biomolecular modeling. Technol Cancer Res Treat 10: 103-112. 
20. Bergholt M, Zheng W, Ho KY, Teh M, Yeoh KG, et al. (2014) Fiberoptic confocal raman spectroscopy for real-time in-vivo diagnosis of dysplasia in barrett's esophagus. Gastroenterology 146: 27-32.
21. Hutchings J, Kendall C, Shepherd N, Barr H, Stone N (2010) Evaluation of linear discriminant analysis for automated Raman histological mapping of esophageal highgrade dysplasia. J Biomed Opt 15: 066015. 